\title{
Zitterbewegung of a Model Universe
}

\author{
R. B. Mann ${ }^{1}$ \\ Department of Physics \& Astronomy, University of Waterloo, \\ Waterloo, Ontario N2L 3G1, Canada \\ G.L. Murphy² \\ St. Paul's Episcopal Church, Akron, Ohio, U.S.A.
}

PACs Numbers: 04.60.+n, 98.80.Dr

January 13, 2019

\begin{abstract}
We investigate the quantum evolution of the metric operators for Bianchi-Type I model universes in the Heisenberg picture in order to remove the need to consider the wave function of the universe and interpret its "spin" variables. The calculation is analogous to that of the Zitterbewegung of the Dirac electron. We consider the behavior of the metric near the classical singularity, and consider the curvature there. Although factor ordering questions preclude the presentation of an unambiguous result for the curvature invariants, it does seem that the classical $t^{-4}$ divergence of the Kretschmann scalar is not removed by quantization.
\end{abstract}

\footnotetext{
${ }^{1}$ rbmann@sciborg.uwaterloo.ca

${ }^{2}$ email: gmurphy@raex.com
} 


\section{Introduction}

The Hamiltonian treatment of simple cosmological models has provided one useful approach to quantum cosmology. In the classical case the canonical equations have solutions which represent solutions of Einstein's field equations, and the hope is that the corresponding quantum equations will represent a full and consistent theory of quantized gravitation[1]. The Hamiltonians for homogeneous universes in general relativity occur in squared form, the simplest example being that for empty Bianchi-Type I spaces with two degrees of freedom:

$$
H^{2}=p_{+}^{2}+p_{-}^{2}
$$

where we take $c$, but not $\hbar$ to be one.

The problem of quantizing a system with such a Hamiltonian already had to be faced in the early days of relativistic quantum theory. The answer which led to the greatest progress was that of Dirac, who extracted the square-root of the corresponding expression for a particle by introducing spinors. With $H^{2}=p^{2}+m^{2}$, one writes

$$
H=\alpha \cdot \mathbf{p}+\beta m
$$

with $(\alpha, \beta)$ a set of anti-commuting $4 \times 4$ matrices. This led, as is well known, to a natural understanding of electron spin and related phenomena.

The same type of procedure can be carried out for cosmological models with a few degrees of freedom. With the Hamiltonian given by (1) we write

$$
H=\sigma_{+} p_{+}+\sigma_{-} p_{-}
$$

where the $\sigma$ 's are $2 \times 2$ Pauli Matrices. Plane wave solutions of the resulting Schrödinger equations are then easily found. But this procedure, while mathematically quite simple, raises interpretative problems. The most obvious difficulty is that the significance of states corresponding to "spin up" and "spin down" for a cosmological model remains obscure[2].

Since this difficulty has to do with interpretation of the state vector, it may be helpful to follow a route that focuses instead on the dynamical variables of the model. Rather than work in the Schrödinger picture, as has usually been done in quantum cosmology, we may deal with the problem in the Heisenberg picture. This approach provided some novel insights into the dynamics of the Dirac electron, and may be expected to do the same for a model universe.

\section{The Zitterbewegung of the Electron}

For the Dirac Hamiltonian (2), the Heisenberg equations of motion give the velocity of the particle as $d x^{i} / d t=\left[x^{i}, H\right] / i \hbar=\alpha^{i}$. Proceeding further, we obtain $i \hbar d \alpha^{i} / d t=-2 \alpha^{i} H-2 p^{i}$, a differential equation with the solution $\alpha^{i}=A^{i} \exp (-2 i H t / \hbar)+p^{i} H^{-1}$, where the Ai are constant. Since $\alpha^{i}=1$ and $p^{i} H^{-1} \leq 1$, the magnitude of the $A^{i}$ must be of order unity. Another integration yields

$$
x^{i}=\left(i \hbar A^{i} / 2\right) \exp (-2 i H t / \hbar) H^{-2}+p^{i} H^{-1} t+a^{i}
$$


with the $a^{i}$ further constants. The second and third terms in (4) represent the motion of a particle that is expected from classical dynamics, motion in a straight line with constant velocity $\mathbf{p} / H$. The first term, on the other hand, represents a quantum oscillation about that classical motion with high frequency $(>2 m / \hbar)$ and an amplitude comparable with the particle's reduced Compton wavelength. This is the Zitterbewegung[3] or "trembling motion". When the Dirac equation is taken to describe a single particle, the peculiarly quantum motion provides one way of thinking about the spin of that particle.

\section{Quantum Cosmology in the Heisenberg Picture: The Metric}

In the cosmological problem, the co-ordinates canonical to $p$ are denoted by $\beta$, and we use a time parameter $\Omega$, which is related to the conventional cosmic time $t$ by $t=(4 \pi R 3 / H) e^{-3 \Omega}$, where $R$ is a constant. (Thus $t \rightarrow 0$ corresponds to $\Omega \rightarrow \infty$.) The diagonal spatial metric is given by

$$
\begin{aligned}
& g_{11}=R^{2} e^{-2 \Omega} \exp \left[2\left(\beta_{+}+\sqrt{3} \beta_{-}\right)\right] \\
& g_{22}=R^{2} e^{-2 \Omega} \exp \left[2\left(\beta_{+}-\sqrt{3} \beta_{-}\right)\right] \\
& g_{33}=R^{2} e^{-2 \Omega} \exp \left[-4 \beta_{+}\right]
\end{aligned}
$$

The classical equations of motion with (1) then give simply $\beta_{ \pm}=\left(p_{ \pm} / H\right) \Omega$ or

$$
\beta_{+}=\Omega \cos \theta, \quad \beta_{-}=\Omega \sin \theta
$$

if $\beta(0)=0$, where $\cos \theta=p_{+} / H$ and $\sin \theta=p_{-} / H$. When these expressions are substituted into (5), we obtain a representation of the well-known Kasner metric[4].

We now move to the corresponding quantum calculation with the Hamiltonian (3). If we use $\sigma_{+} \sigma_{-}+\sigma_{+} \sigma_{-}=0, \sigma_{+}^{2}=\sigma_{-}^{2}=1$, the Heisenberg equations of motion give us, in analogy with (4),

$$
\beta_{ \pm}=\left(i \hbar B_{ \pm} / 2\right) \exp (-2 i H \Omega / \hbar) H^{-2}+p_{ \pm} H^{-1} \Omega+C_{ \pm}
$$

Here the $B$ 's and $C$ 's are constant $2 \times 2$ matrices.

It is tempting to think that the $C$ 's can essentially be ignored. A similar constant of integration representing the initial position of a particle can often be dropped in elementary mechanics by just defining the initial position to be at the origin of coordinates. But in quantum theory matters are different, as we can see by considering the elementary problem of the motion of a non-relativistic free particle with $H=p^{2} / 2 m$.

The Heisenberg equations of motion easily give $p=$ constant and $q=(p / m) t+q_{0}$. We might be tempted to ignore the constant $q_{0}$ but it cannot vanish because the commutation relations between $q$ and $p$ require that $\left[q_{0}, p\right]=i \hbar$.

Having to retain the $C$ 's as additive constants might not seem to be a serious problem. But in our case the required exponentiation of the $\beta$ 's in the metric mean that we will have multiplicative constant operators which do not commute with the $p$ 's in the metric. This will complicate the quantum calculations. 
The metric components, obtained by substituting (7) into (5), are now operators which carry the time-dependence of the model. They are

$$
\begin{aligned}
g_{11} & =\left(\exp D_{1}\right) R^{2} \exp \left[i \hbar\left(B_{+}+\sqrt{3} B_{-}\right) \exp (-2 i H \Omega / \hbar) H^{-1}\right] \exp [2(\cos \theta+\sqrt{3} \sin \theta-1) \Omega] \\
g_{22} & =\left(\exp D_{2}\right) R^{2} \exp \left[i \hbar\left(B_{+}-\sqrt{3} B_{-}\right) \exp (-2 i H \Omega / \hbar) H^{-1}\right] \exp [2(\cos \theta-\sqrt{3} \sin \theta-1) \Omega](8) \\
g_{33} & =\left(\exp D_{3}\right) R^{2} \exp \left[-2 i \hbar B+\exp (-2 i H \Omega / \hbar) H^{-1}\right] \exp [-2 \Omega(1+2 \cos \theta)]
\end{aligned}
$$

where the exponentiated $D$ 's are the multiplicative constant operators noted above.

In terms of the cosmic time $t$ we have

$$
\begin{aligned}
& g_{11}=\left(\exp D_{1}\right) R^{2} \exp \left[i \hbar\left(B_{+}+\sqrt{3} B_{-}\right)(H t / S)^{2 i H / 3 \hbar} H^{-1}\right](H t / S)^{2(\cos \theta+\sqrt{3} \sin \theta-1) / 3} \\
& g_{22}=\left(\exp D_{2}\right) R^{2} \exp \left[i \hbar\left(B_{+}-\sqrt{3} B_{-}\right)(H t / S)^{2 i H / 3 \hbar} H^{-1}\right](H t / S)^{2(\cos \theta-\sqrt{3} \sin \theta-1) / 3} \\
& g_{33}=\left(\exp D_{3}\right) R^{2} \exp \left[2 i \hbar B_{+}(H t / S)^{2 i H / 3 \hbar} H^{-1}\right](H t / S)^{(1+2 \cos \theta)}
\end{aligned}
$$

where $S=4 \pi R^{3}$. The third exponential factors in (8) and (9) give the classical Kasner behavior, while the preceding factors contain the distinctive quantum mechanical behavior. It should be noted, though, that $\cos \theta$ and $\sin \theta$, now defined by (6) as $p_{+} H^{-1}$ and $p_{-} H^{-1}$ respectively, are operators, so that the expressions for $g_{i j}$ do not split cleanly into "classical" and "quantum" factors.

Since $H^{2}=p^{2}=p_{+}^{2}+p_{-}^{2}$ we have $\exp (a H)=\cosh (a p)+(H / p) \sinh (a p)$. If we use this result in (7) we find

$$
\beta_{ \pm}=\left[\left(\hbar / 2 p^{2}\right) B_{ \pm} \sin \left(\frac{2 \Omega p}{\hbar}\right)+p_{ \pm} \Omega / p\right](H / p)+\left(i \hbar / 2 p^{2}\right) B_{ \pm} \cos \left(\frac{2 \Omega p}{\hbar}\right)+C_{ \pm}
$$

The metric components can then be written as

$$
g_{i i}=\left(\exp D_{i}\right) R^{2} \exp \left[\lambda_{i}(t)+\gamma_{i}(t) H / p\right]
$$

where

$$
\begin{aligned}
& \lambda_{ \pm}=-2 \Omega+i\left[\hbar\left(B_{+} \pm \sqrt{3} B_{-}\right) / p^{2}\right] \cos \left(\frac{2 \Omega p}{\hbar}\right) \\
& \lambda_{3}=-2 \Omega-2 i\left(\hbar B_{+} / p^{2}\right) \cos \left(\frac{2 \Omega p}{\hbar}\right) \\
& \gamma_{ \pm}=\left(\hbar / p^{2}\right)\left(B_{+} \pm \sqrt{3} B_{-}\right) \sin \left(\frac{2 \Omega p}{\hbar}\right)+2\left(p_{+} \pm \sqrt{3} p_{-}\right) \Omega / p \\
& \gamma_{3}=-2\left[\left(\hbar / p^{2}\right) B_{+} \sin \left(\frac{2 \Omega p}{\hbar}\right)+2 p_{+} \Omega / p\right]
\end{aligned}
$$

with $D_{1}=2\left(C_{+}+\sqrt{ } 3 C_{-}\right), D_{2}=2\left(C_{+}-\sqrt{ } 3 C_{-}\right)$, and $D_{3}=-4 C_{+}$. (We also have dropped the summation convention.) 
For brevity we put $\phi_{i}=\lambda_{i}+\gamma_{i} H / p$ so that $g_{i i}=\exp \left(D_{i}\right) R^{2} \exp \left(\phi_{i}\right)$. The contravariant components $g^{i i}=R^{-2} \exp \left(-\phi_{i}\right) \exp \left(-D_{i}\right)$ will then be the correct inverse of $g_{i i}$.

The temporal evolution of the metric is now rather complicated. The quantum feature par excellence is the fact that $B$ and $H$ are operators which can be represented by $2 \times 2$ matrices in the present model. But we should also note that the simple complex exponential or circular functions which describe the Zitterbewegung of a particle are now themselves the arguments of the exponential functions which give the metric in (8) and (9). As $t \rightarrow 0$ and $\Omega \rightarrow \infty$, the magnitude of the metric components will oscillate an infinite number of times.

\section{Quantum Cosmology in the Heisenberg Picture: The Curvature}

In order to investigate the behavior of space-time near $t=0(\Omega=\infty)$, the true singularity of the classical model, we must of course examine not simply the metric but the curvature.

In the classical case we can use the cosmic time $t$ and write the metric corresponding to (9) as $g_{00}=-1, g_{a a}=t^{2 s_{a}}$ with appropriate scaling. The non-vanishing components of the Riemann tensor are given by $R_{a 0 a 0}=-\Gamma_{0 a a, 0}+\Gamma_{a a 0} \Gamma_{0 a a}=-s_{a}\left(s_{a}-1\right) t^{2 s_{a}-2}$ and the Kretschmann scalar $K=R_{\alpha \beta \gamma \delta} R^{\alpha \beta \gamma \delta}$

$$
K=-16 \frac{s_{1} s_{2} s_{3}}{t^{4}}
$$

which diverges as $t \rightarrow 0$.

The quantum theory is more complicated and there is considerable ambiguity because of the non-commutativity of operators. There are three ways in which this complicates matters. First, there is the factor-ordering problem, which always arises when a transition is made from a classical to a quantum theory. Secondly, the operator scale factors in the metric which we noted above must be taken into account. Finally, we have to be aware that the $B$ 's must have an algebraic structure ensuring that $\sigma$ 's (which now in the Heisenberg picture are operators) have the correct algebra.

If we choose the usual order of factors for the Christoffel symbols we obtain

$$
\Gamma_{m 0 n}=\frac{1}{2} \delta_{m n} \phi_{m}^{\prime}, \quad 0 m n=\frac{1}{2} R_{0}^{2}\left(\exp D_{m}\right) \delta_{m n} \phi_{m}^{\prime}\left(\exp \phi_{m}\right),
$$

where a prime denotes the time derivative. We then calculate curvature components with the ordering of factors given by

$$
R_{\mu \nu \kappa}^{\lambda}=\Gamma_{\mu \nu, \kappa}^{\lambda}-\Gamma_{\mu \kappa, \nu}^{\lambda}+\Gamma_{\mu \nu}^{\sigma} \Gamma_{\kappa \sigma}^{\lambda}-\Gamma_{\mu \kappa}^{\sigma} \Gamma_{\nu \sigma}^{\lambda}
$$

It would be desirable, as we will see below, for factors to be ordered in such a way that all occurrences of $\exp D_{m}$ in the curvature occur to the left of other factors. Unfortunately this would require a different ordering of factors for different components, so that such a result would not be covariant. Instead of trying to indicate all the possibilities we will simply follow the prescription in (13) and write the results as follows. 


$$
\begin{aligned}
R_{0 n 0}^{m} & =\frac{1}{2} \delta_{m n}\left(\phi_{m}^{\prime \prime}+\phi_{m}^{\prime 2}\right) \\
R_{m n 0}^{0} & =\frac{1}{2} \exp D_{m}\left(R_{0}^{2}\right) \delta_{m n}\left[\phi_{m}^{\prime \prime}+\frac{1}{2} \phi_{m}^{\prime 2}\right] \exp \phi_{m}+C . T \\
R_{m n k}^{l} & =\frac{1}{2} \exp D_{m}\left(R_{0}^{2}\right)\left(\delta_{m n} \delta_{k l}-\delta_{m k} \delta_{n l}\right) \phi_{m}^{\prime} \phi_{l}^{\prime}+C . T
\end{aligned}
$$

Here C.T. denotes "commutator terms," distinctively quantum mechanical expressions involving $\hbar$, which result from switching the order of factors containing $D$ 's (and thus $C$ 's) and $p$ 's.

We then find that the mixed Ricci tensor components are

$$
\begin{aligned}
R_{0}^{0}= & \left(\frac{2}{3 p^{2} t^{2}}\right)\left\{B^{2}\left[\cos ^{2}\left(\frac{2 \Omega p}{\hbar}\right)-\sin ^{2}\left(\frac{2 \Omega p}{\hbar}\right)\right]\right. \\
& \left.+2 \mathbf{p B} \cos \left(\frac{2 \Omega p}{\hbar}\right)+2 i \sin \left(\frac{2 \Omega p}{\hbar}\right)\left[B^{2} \cos \left(\frac{2 \Omega p}{\hbar}\right)+\mathbf{p B}\right] \frac{H}{p}\right\}+ \text { C.T. } \\
R_{1}^{1}= & -2 i\left(\frac{B_{+}+\sqrt{3} B_{-}}{9 \hbar t^{2}}\right)\left[\cos \left(\frac{2 \Omega p}{\hbar}\right)+i \sin \left(\frac{2 \Omega p}{\hbar}\right) \frac{H}{p}\right]+\text { C.T } \\
R_{2}^{2}= & -2 i\left(\frac{B_{+}-\sqrt{3} B_{-}}{9 \hbar t^{2}}\right)\left[\cos \left(\frac{2 \Omega p}{\hbar}\right)+i \sin \left(\frac{2 \Omega p}{\hbar}\right) \frac{H}{p}\right]+\text { C.T } \\
R_{3}^{3}= & 4 i\left(\frac{B_{+}}{9 \hbar t^{2}}\right)\left[\cos \left(\frac{2 \Omega p}{\hbar}\right)+i \sin \left(\frac{2 \Omega p}{\hbar}\right) \frac{H}{p}\right]+\text { C.T }
\end{aligned}
$$

Because of cancellations the Ricci scalar $R$ is simply equal to $R_{0}^{0}$. We have written $\mathbf{p B}=p_{+} B_{+}+$ $p_{-} B_{-}$and $B^{2}=B_{+}^{2}+B_{-}^{2}$. In the calculation of the mixed Ricci components, all occurrences of the commutator of $B_{+}$and $B_{-}$cancel out.

Calculation of the Kretschmann scalar is now considerably more complicated, even if the problems of factor ordering are ignored. In addition, the need for four factors of metric components to lower and raise indices introduces a great many more choices for their ordering, and the noncommutativity of the Bs can no longer be ignored. The full expression for $\mathrm{K}$ with the choices we have made for factor ordering is quite lengthy. It can be written as

$$
K=K_{0}+K_{1} \frac{H}{p}
$$

where

$$
\begin{aligned}
K_{0}= & \frac{4}{27 \hbar^{2} t^{4}}\left[8 B^{2}\left(1-2 \cos ^{2}\left(\frac{2 \Omega}{\hbar} p\right)\right)+5 \hbar^{2}\right]+\frac{32 \sin \left(\frac{2 \Omega}{\hbar} p\right)}{27 \hbar t^{4} p}\left[2 B^{2} \cos \left(\frac{2 \Omega}{\hbar} p\right)+\vec{p} \cdot \vec{B}\right] \\
& -\frac{8}{27 \hbar t^{4} p^{2}}\left[-3 \hbar\left(B^{2}-p^{2}\right)+8 i\left(B_{+}^{2} p_{+}-B_{-}^{2} p_{+}-2 p_{-} B_{+} B_{-}\right)\right]
\end{aligned}
$$




$$
\begin{aligned}
& +\frac{16 i}{27 \hbar t^{4} p^{2}} \cos \left(\frac{2 \Omega}{\hbar} p\right)\left\{3 i \hbar \vec{p} \cdot \vec{B}-6 B_{+}\left(B_{+}+\sqrt{3} B_{-}\right)\left(B_{+}-\sqrt{3} B_{-}\right)+2\left(p_{+}^{2} B_{+}-p_{-}^{2} B_{+}-2 B_{-} p_{+} p_{-}\right)\right\} \\
& +\frac{16 i \cos ^{2}\left(\frac{2 \Omega}{\hbar} p\right)}{27 \hbar t^{4} p^{2}}\left[3 i \hbar B^{2}+8\left(B_{+}^{2} p_{+}-B_{-}^{2} p_{+}-2 p_{-} B_{+} B_{-}\right)\right] \\
& -\frac{128 i \cos ^{3}\left(\frac{2 \Omega}{\hbar} p\right)}{27 \hbar t^{4} p^{2}} B_{+}\left(B_{+}+\sqrt{3} B_{-}\right)\left(B_{+}-\sqrt{3} B_{-}\right) \\
& -\frac{128 i \sin \left(\frac{2 \Omega}{\hbar} p\right) \cos ^{2}\left(\frac{2 \Omega}{\hbar} p\right)}{27 t^{4} p^{3}}\left(B_{+}\left(B_{+}+\sqrt{3} B_{-}\right)\left(B_{+}-\sqrt{3} B_{-}\right)\right) \\
& -\frac{64 i \sin \left(\frac{2 \Omega}{\hbar} p\right)}{9 t^{4} p^{3}} \cos \left(\frac{2 \Omega}{\hbar} p\right)\left(B_{+}^{2} p_{+}-B_{-}^{2} p_{+}-2 p_{-} B_{+} B_{-}\right) \\
& -\frac{32 i \sin \left(\frac{2 \Omega}{\hbar} p\right)}{27 t^{4} p^{3}}\left(-6 B_{-} p_{+} p_{-}+3 B_{+} B_{-}^{2}+3 B_{+} p_{+}^{2}-3 B_{+} p_{-}^{2}-B_{+}^{3}\right) \\
& +\frac{32}{3 t^{4} p^{4}} B^{4} \cos ^{4}\left(\frac{2 \Omega}{\hbar} p\right)+\frac{64}{3 t^{4} p^{4}} \cos ^{3}\left(\frac{2 \Omega}{\hbar} p\right)\left(B_{-}^{3} p_{-}+B_{+} B_{-}^{2} p_{+}+B_{+}^{3} p_{+}+B_{+}^{2} B_{-} p_{-}\right) \\
& +\frac{16}{3 t^{4} p^{4}} \cos ^{2}\left(\frac{2 \Omega}{\hbar} p\right)\left(B_{+}^{2} p_{-}^{2}+3 B_{-}^{2} p_{-}^{2}-2 B^{4}+3 B_{+}^{2} p_{+}^{2}+4 B_{-} p_{-} B_{+} p_{+}-4 B_{+}^{2} B_{-}^{2}+B_{-}^{2} p_{+}^{2}\right) \\
& +\frac{16}{3 t^{4} p^{4}} \cos ^{2}\left(\frac{2 \Omega}{\hbar} p\right)\left(p_{-}^{3} B_{-}+p_{+}^{3} B_{+}+p_{+} p_{-}^{2} B_{+}-3 B_{+}^{2} B_{-} p_{-}-3 B_{+}^{3} p_{+}-3 B_{-}^{3} p_{-}-3 B_{+} B_{-}^{2} p_{+}+B_{-} p_{+}^{2} p_{-}\right) \\
& +\frac{4}{3 t^{4} p^{4}}\left(p^{4}+B^{4}-2 B_{+}^{2} p_{-}^{2}-6 B_{+}^{2} p_{+}^{2}-6 B_{-}^{2} p_{-}^{2}-8 B_{-} p_{-} B_{+} p_{+}-2 B_{-}^{2} p_{+}^{2}\right)
\end{aligned}
$$

and

$$
\begin{aligned}
K_{1}= & -\frac{64 i}{27 t^{4} \hbar^{2}} \sin \left(\frac{2 \Omega}{\hbar} p\right) \cos \left(\frac{2 \Omega}{\hbar} p\right) B^{2}+\frac{32 i}{27 t^{4} \hbar p}\left(B^{2}\left[1-2 \cos ^{2}\left(\frac{2 \Omega}{\hbar} p\right)\right]-2 \vec{p} \cdot \vec{B} \cos \left(\frac{2 \Omega}{\hbar} p\right)\right) \\
& -\frac{128 \sin \left(\frac{2 \Omega}{\hbar} p\right) \cos ^{2}\left(\frac{2 \Omega}{\hbar} p\right)}{27 \hbar t^{4} p^{2}}\left(B_{+}\left(B_{+}+\sqrt{3} B_{-}\right)\left(B_{+}-\sqrt{3} B_{-}\right)\right) \\
& -\frac{16 \sin \left(\frac{2 \Omega}{\hbar} p\right) \cos \left(\frac{2 \Omega}{\hbar} p\right)}{27 \hbar t^{4} p^{2}}\left(3 i \hbar B^{2}+8\left(B_{+}^{2} p_{+}-B_{-}^{2} p_{+}-2 p_{-} B_{+} B_{-}\right)\right) \\
& -\frac{16 \sin \left(\frac{2 \Omega}{\hbar} p\right)}{27 \hbar t^{4} p^{2}}\left(3 i \hbar \vec{p} \cdot \vec{B}+2\left(p_{+}^{2} B_{+}-p_{-}^{2} B_{+}-2 p_{-} p_{+} B_{-}\right)+6 B_{+} B_{-}^{2}-2 B_{+}^{3}\right) \\
& -\frac{128 \cos ^{3}\left(\frac{2 \Omega}{\hbar} p\right)}{27 t^{4} p^{3}}\left(B_{+}\left(B_{+}+\sqrt{3} B_{-}\right)\left(B_{+}-\sqrt{3} B_{-}\right)\right)-\frac{64 \cos ^{2}\left(\frac{2 \Omega}{\hbar} p\right)}{9 t^{4} p^{3}}\left(B_{+}^{2} p_{+}-B_{-}^{2} p_{+}-2 p_{-} B_{+} B_{-}\right) \\
& -\frac{32 \cos \left(\frac{2 \Omega}{\hbar} p\right)}{9 t^{4} p^{3}}\left(3 B_{+} B_{-}^{2}-B_{+}^{3}+\left(p_{+}^{2} B_{+}-p_{-}^{2} B_{+}-2 p_{-} p_{+} B_{-}\right)\right) \\
& -\frac{32}{27 t^{4} p^{3}}\left(6 B_{+} B_{-} p_{-}+3 B_{-}^{2} p_{+}+p_{+}^{3}-3 B_{+}^{2} p_{+}-3 p_{-}^{2} p_{+}\right) \\
& +\frac{32 i \sin \left(\frac{2 \Omega}{\hbar} p\right) \cos ^{3}\left(\frac{2 \Omega}{\hbar} p\right)}{3 t^{4} p^{2}} B^{4}+\frac{64 i \sin \left(\frac{2 \Omega}{\hbar} p\right) \cos ^{2}\left(\frac{2 \Omega}{\hbar} p\right)}{3 t^{4} p^{2}}\left(p_{+} B_{+} B_{-}^{2}+B_{-}^{3} p_{-}+B_{+}^{3} p_{+}+3 B_{+}^{2} B_{-} p_{-}\right)
\end{aligned}
$$




$$
\begin{aligned}
& +\frac{16 i \sin \left(\frac{2 \Omega}{\hbar} p\right) \cos \left(\frac{2 \Omega}{\hbar} p\right)}{3 t^{4} p^{2}}\left(-2 B_{+}^{2} B_{-}^{2}+4 B_{+} B_{-} p_{-} p_{+}+3 p_{-}^{2} B_{-}^{2}+3 p_{+}^{2} B_{+}^{2}+B_{+}^{2} p_{-}^{2}-B_{-}^{4}+B_{-}^{2} p_{+}^{2}-B_{+}^{4}\right) \\
& +\frac{16 i \sin \left(\frac{2 \Omega}{\hbar} p\right)}{3 t^{4} p^{2}}\left(-p_{+} B_{+}^{3}+p_{-}^{2} p_{+} B_{+}+p_{+}^{3} B_{+}+p_{+}^{2} p_{-} B_{+}-B_{+} B_{-}^{2} p_{+}-B_{+}^{2} B_{-} p_{-}+B_{-} p_{-}^{3}-B_{-}^{3} p_{-}\right)
\end{aligned}
$$

The terms $\tilde{K}$ that do not involve any distinctively quantum factors, and that are not affected by factor ordering complications, can be written as

$$
\tilde{K}=\frac{8}{27 t^{4}}\left\{10+\left[4\left(\frac{3 p_{-}^{2}-p_{+}^{2}}{p^{3}}\right)\right] p_{+} \frac{H}{p}\right\}
$$

This is identical with the classical result given by (12). This means that while the magnitude of this curvature invariant will undergo oscillations of increasing rapidity as $t \rightarrow 0$, there will be an overall unbounded increase beyond any bounds according to the same $t^{-4}$ law that obtains in the classical case. The leading divergence of the curvature is not removed by quantization.

There are, of course, profound differences between classical and quantum curvatures in other regards. As is well known, the special case of the Kasner metric with $s_{1}=s_{2}=0$ and $s_{3}=1$ in the classical theory is simply flat space-time. This is no longer so in quantum theory because of the additional exponential factors in the expressions (9) for the metric.

Use of the Heisenberg picture thus gives some novel insight into the quantum dynamics of the simplest model universe of the Bianchi type, and shows in particular that the initial singularity is not avoided by quantization. There are no questions about boundary conditions on a wave function or the interpretation of it to cloud this result. Unfortunately the procedure followed here cannot be extended immediately to more complicated models. Just as straightforward integration of the equations of motion for the Dirac electron works only for a free particle and not when an external potential is present, the curvature term which would be added to right side of (1) frustrates attempts to follow the same procedure for spaces of other Bianchi types.

In a sense our result is not too surprising. When we solve the Dirac eqn for a particle we recover the classical motion of the particle together with the distinctive Zitterbewegung effects. In the cosmological case a similar thing happens - we recover the classical cosmological motion corrected by quantum effects. But in this case the classical solution is divergent, and the question of interest is whether or not the quantum effects can cancel this divergence. In particular, does the expectation value of the Kretschmann scalar blow up as $t \rightarrow 0$ in the quantum case as it does in the classical one?

We have not been able to answer this question definitively because of the huge number of possible factor orderings in $K$ that arise in different ways. The most straightforward way of writing $K$ does give a divergent result, and this may suggest that hopes for an elimination of the initial cosmological singularity by quantum theory will be disappointed. But it is not impossible that some choice of factor ordering will eliminate this divergence.

Use of the Heisenberg picture thus gives some novel insight into the quantum dynamics of the simplest model universe of the Bianchi type, and shows in particular that the initial singularity is not avoided by quantization. There are no questions about boundary conditions on a wave function or the interpretation of it to cloud this result. Unfortunately the procedure followed here cannot be extended immediately to more complicated models. Just as straightforward integration 
of the equations of motion for the Dirac electron works only for a free particle and not when an external potential is present, a term involving the 3-space curvature term which would be added to right side of (1) frustrates attempts to follow the same procedure for spaces of other Bianchi types. The problem would, of course, be even more difficult for nonhomogeneous universes or other space-times[5].

\section{Acknowledgments}

This work was supported in part by the Natural Sciences and Engineering Research Council of Canada.

\section{References}

[1] B. DeWitt, Phys. Rev. 160, 1113 (1967): C. Misner, "Classical and Quantum Dynamics of a Closed Universe" M. Carmeli, S.I. Fickler and L. Witten, Relativity (Plenum, New York, 1970), pp.55-79: M. Ryan, Hamiltonian Cosmology (Springer-Verlag, New York, 1972). The notation of the latter monograph is used in this paper.

[2] G.L. Murphy, Found. Phys. 4, 351, (1984).

[3] E. Schrödinger, Sitzungb. d. Berlin. Akad. XXIV, 418 (1930): P.A.M. Dirac, The Principles of Quantum Mechanics, 4th ed. (Clarendon, Oxford, 1958), pp.261-263.

[4] E. Kasner, Am. J. Math. 43, 217 (1921).

[5] M. Ryan, Hamiltonian Cosmology (Springer-Verlag, New York, 1972) p 25. 